\title{
Igf2-H19, an imprinted tandem gene, is an important regulator of embryonic development, a guardian of proliferation of adult pluripotent stem cells, a regulator of longevity, and a 'passkey' to cancerogenesis
}

\author{
Mariusz Z. Ratajczak ${ }^{1,2}$ \\ ${ }^{1}$ Stem Cell Biology Program at the James Graham Brown Cancer Center, University of Louisville, \\ Louisville, Kentucky, USA \\ ${ }^{2}$ Department of Physiology, Pomeranian Medical University, Szczecin, Poland
}

\begin{abstract}
The insulin-like growth factor-2 (Igf)-H19 locus encodes important paternally imprinted genes that govern normal embryonic development. While Igf-2 encodes IGF2, which is an autocrine/paracrine mitogen, transcription of $H 19$ gives rise to non-coding mRNA that is a precursor of several microRNAs (miRNAs) that negatively affect cell proliferation. The proper imprinting of a differentially methylated region (DMR) within this locus, with methylation of the paternal chromosome and a lack of methylation on the maternal chromosome, regulates expression of both of these genes so that $\operatorname{Ig} 2$ is transcribed only from the paternal chromosome and $H 19$ only from the maternal chromosome. There is growing evidence that this 'Yin-Yang' locus regulates embryonic development. Furthermore, recent evidence indicates that erasure of imprinting (hypomethylation) of the Igf- $-H 19$ locus on both chromosomes, which leads to downregulation of Igf2 and upregulation of H19 expression, plays an important role in regulating quiescence of pluripotent stem cells in adult organisms, and may be involved in the regulation of lifespan. In contrast, hypermethylation of this locus on both chromosomes (loss of imprinting) results in Igf 2 overexpression and is observed in several malignancies. In this review, we will discuss the biological consequences of changes in Igf2-H19 expression. (Folia Histochemica et Cytobiologica 2012, Vol. 50, No. 2, 171-179)
\end{abstract}

Key words: imprinting, Igf2, H19, VSELs, longevity, cancerogenesis

\section{Introduction}

Mammalian development requires proper gene dosage, which is enabled by the imprinting of genes so that a single parental allele is expressed. In other words, genomic imprinting is an epigenetic program that ensures the parent-of-origin-specific monoallelic transcription of imprinted genes and results in in-

Correspondence address: M.Z. Ratajczak,

Stem Cell Institute at James Graham Brown Cancer Center, University of Louisville,

500 S. Floyd Street, Rm. 107 Louisville, KY 40202, USA; tel: + 502852 1788, fax: + 502852 3032;

e-mail: mzrata01@louisville.edu tracellular expression of imprinted genes from only one of the two paternal chromosomes, derived either from the mother or the father [1-5].

Overall, there are $\sim 100$ paternally imprinted genes in the mammalian genome (expressed from the maternal or paternal chromosome only) that play an important role in embryonal development. Imprinted genes play a crucial role in embryogenesis, fetal growth, behavioral development, the totipotential state of the zygote, and pluripotency of developmentally early stem cells [4]. On the other hand, erasure of genomic imprints is one of the mechanisms that prevent some of the pluripotent stem cells (e.g. primordial germ cells [PGCs] and very small embryon- 
ic-like stem cells [VSELs]) from uncontrolled proliferation and teratoma formation [6-13].

The expression of imprinted genes is regulated by the imposition of epigenetic marks by DNA methylation within differentially methylated regions (DMRs), which are CpG-rich cis-elements within their loci [1-4]. These epigenetic marks imposed on DMRs in the female germline act on the promoters of imprinted genes, which results in the heritable repression of the maternal chromosomes. In contrast, the imposition of epigenetic marks by methylation of the chromosomes in the male germline does not occur at the promoters, but rather within the intergenic regions (i.e. between the Igf 2 and $H 19$ genes). Imprinted genes, in general, are highly expressed during embryogenesis, and many of them are subsequently downregulated after birth. Most imprinted genes are methylated on maternally derived chromosomes, and only four: Igf2-H19, RasGrf1, Dlk1/Dio3, and Zdbf2, are methylated on paternally derived chromosomes [1-4]. In this review, we will focus on the biological consequences of imprinting within the DMR for Igf2-H19.

The Igf2-H19 tandem gene is paternally imprinted in humans as well as in mice and, as mentioned above, the regulatory DMR for this gene is methylated on the paternal, and erased on the maternal, chromosome [1-4]. Figure 1 shows a schematically simplified structure for this locus. The filled lollypops at the DMR regulatory region of the paternal chromosome depict methylation, and open lollypops on the maternal chromosome indicate lack of methylation. If the DMR is methylated, it cannot bind the regulatory DNA-binding zinc finger insulator protein, CTCF, which establishes a functional boundary between the $I g f 2$ and $H 19$ coding regions. The binding of CTCF has immediate consequences for the expression of these loci. Since the expression of both $I g f 2$ and $H 19$ is regulated from a 3' distal enhancer (shown as a green box), the presence of CTCF bound to the DMR at the maternal locus prevents transcription of Igf2, and in this situation, only $H 19$ is transcribed to RNA. In contrast, the presence of a methylated DMR on the paternal chromosome prevents binding of CTCF, and in this situation, the 3' distal enhancer promotes transcription of mRNA from the Igf2 locus. This ensures a proper balance in the expression of both genes (Figure 2, upper panel).

As discussed in this review, the methylation of DMRs within the Igf2-H19 locus is erased in pluripotent stem cells, such as PGCs [6-11] and VSELs [12, 13] (Figure 2, middle panel). This epigenetic modification of Igf2-H19 is one of the mechanisms responsible for keeping these cells quiescent (low expression of Igf 2 and overexpression of H19) and prevents them from uncontrolled proliferation and teratoma formation. In contrast, hypermethylation of DMRs within Igf2-H19 on both maternally and paternally derived chromosomes, which is called 'loss of imprinting', is observed in several malignancies and results in high expression of $\operatorname{Ig} 2$ (Figure 2, lower panel). Loss of imprinting at this locus, as will be discussed later, could also result from perturbation of other mechanisms that regulate the proper Igf2:H19 expression ratio in these cells (e.g.downregulation of CTCF expression) [14].

In this review, we will discuss the three possibilities (normal somatic imprinting, erasure of imprinting, and loss of imprinting) as epigenetic changes in methylation of DMRs for the Igf2-H19 'master locus' that affect several biological processes, such as proper embryogenesis, proliferation of pluripotent stem cells, senescence, and tumorogenesis.

\section{Biological role of proper somatic imprinting of the Igf2-H19 tandem gene}

The tandem Igf2-H19 locus is located on chromosome $11 \mathrm{p} 15$ in humans and chromosome 7 in mice. While the Igf2 locus encodes insulin-like growth factor-2 (IGF2), which is a growth-promoting peptide hormone highly expressed during embryogenesis, the $\mathrm{H} 19$ locus encodes a non-coding RNA (ncRNA) that gives rise to several microRNAs (miRNAs) that have opposite effects on cell proliferation [15]. The dual YinYang function of this master locus is relevant to several biological functions, including normal fetal development, and the properly balanced expression of Igf2 and H19 is crucial for proper embryogenesis (Figure 2 upper panel). Imprinting at the Igf2-H19 locus is one of the major factors preventing parthenogenetic development in mammals, and the biological importance of this locus is demonstrated by the additional steps necessary for the creation of viable bimaternal mice derived from two female sets of chromosomes [1-4]. These mice are created by combining two haploid nuclei, one from non-growing and the other from fully growing oocytes, into a diploid 'bimaternal zygote'. Since female chromosomes have unmethylated DMRs for Igf2-H19 (Figure 1), the crucial step is appropriate genetic modulation of the proper expression of $I g f 2$ from one of the maternally derived sets of chromosomes [6-13].

The role of imprinted genes in development, including the master tandem gene Igf2-H19, has been explained by the parent-offspring conflict hypothesis. While during pregnancy, the father, through proper expression of paternally imprinted genes (i.e. by expression of Igf 2 and suppression of H19), contrib- 

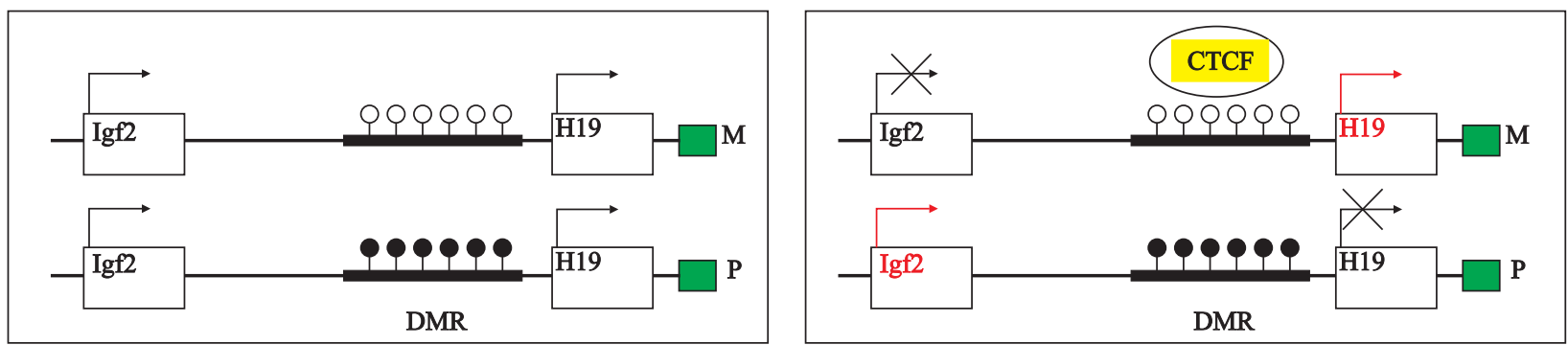

Figure 1. Regulation of expression of the Igf2-H19 tandem gene. Left panel - Igf2 and H19 coding regions are separated by a differentially methylated region (DMR) that is methylated (as shown by filled lollypops) on the paternal chromosome and unmethylated (open lollypops) on the maternal chromosome. Expression of both genes is regulated by a 3' distal enhancer, depicted in green. Right panel - methylation of the DMR on the paternal chromosome prevents binding of CTCF insulator protein and allows activation of the $I g f 2$ promoter by the distal enhancer and transcription of $I g f 2$ mRNA from the paternal chromosome (red arrow). In contrast, since the DMR is unmethylated on the maternal chromosome, it binds CTCF, and this prevents activation of the $I g f 2$ promoter by the distal enhancer. As a result, only $H 19$ mRNA is transcribed from the maternal chromosome (red arrow)

Proper somatic imprint

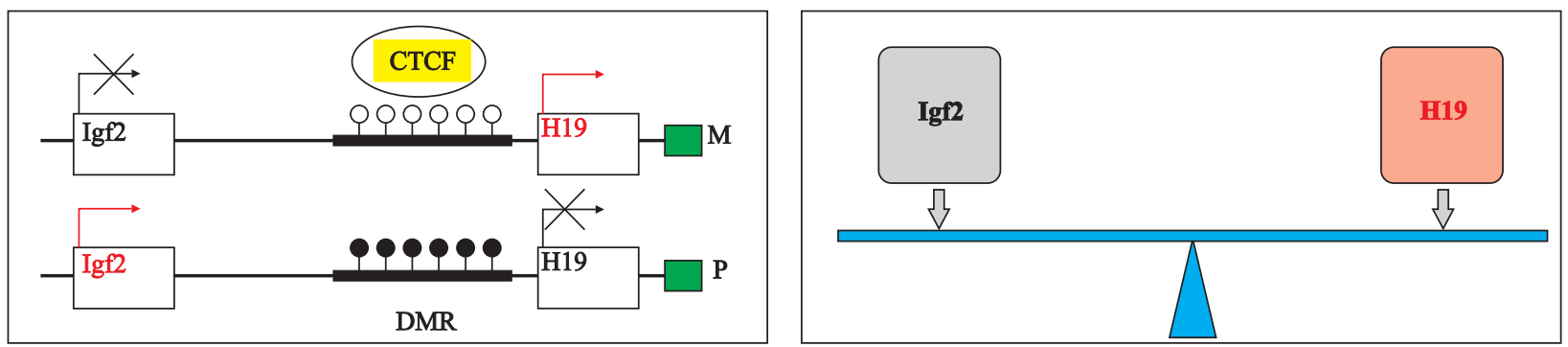

Erasure of imprint

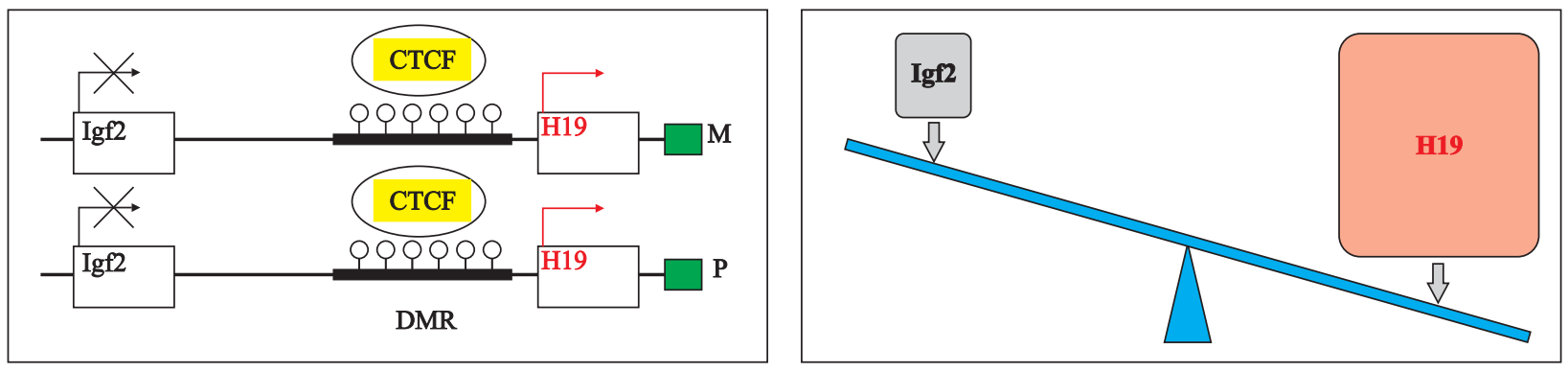

Loss of imprint

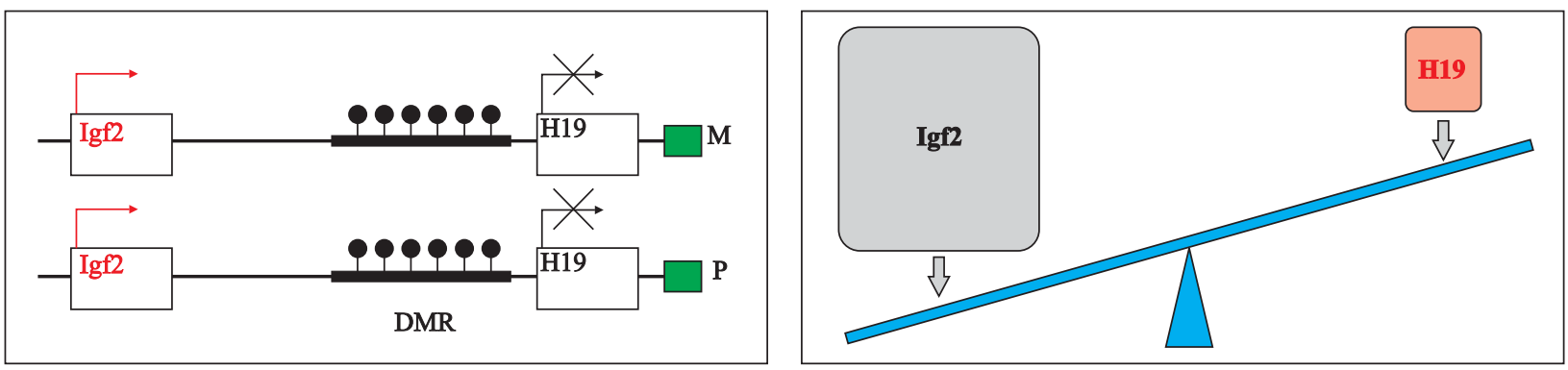

Figure 2. Changes in methylation state of DMRs and their impact on Igf 2 and H19 expression. Upper panel — normal somatic imprint observed in all somatic cells, which results, as described in Figure 1, in properly balanced expression of Igf 2 from the paternal chromosome and $H 19$ from the maternal chromosome (red arrows). Middle panel - erasure of imprinting at the Igf2-H19 locus as seen in primordial germ cells (PGCs) and pluripotent stem cells residing post-developmentally in adult tissues (VSELs). DMRs on both the paternal and maternal chromosomes are engaged by the CTCF insulator protein, and thus only H19 mRNA is transcribed (red arrows), contributing to the quiescent state of cells (lacking autocrine IGF2). Lower panel - loss of imprinting at the Igf2-H19 locus as seen in tumor cells from several types of cancer (e.g. rhabdomyosarcoma, nephroblastoma, and gastrointestinal tumors). Since both DMRs are methylated, the insulator protein CTCF cannot bind to the DNA and the distal enhancer stimulates transcription of mRNA for IGF2 from both chromosomes (red arrows). Cells that have this epigenetic change are under autocrine IGF2 stimulation 
utes to body size and muscle mass of the developing fetus, and wants the mother to devote as much of her resources as possible towards the growth of his offspring, the mother wants to conserve as much of her resources as possible towards future births (without compromising the health of the fetus she is carrying) by epigenetic modulation of genes by maternal imprinting marks (i.e. to achieve suppression of $\operatorname{Ig} f 2$ and expression of H19) [4].

It is well known that IGF2 is secreted from cells in an autocrine or paracrine manner and exerts its biological effects after binding to the insulin-like growth factor-1 (IGF1) receptor (IGF-1R). On the other hand, IGF2 may also bind to non-signaling insulin-like growth factor-2 receptor (IGF-2R), which is a mannose 6-phosphate receptor that acts as a decoy receptor for IGF2 and prevents its signaling through IGF-1R [17]. Overall, the IGF2 level is high in humans during embryogenesis and in postnatal life may be produced and secreted by some tumors, causing their proliferation and hypoglycemia as part of the paraneoplastic syndrome. The loss of imprinting at the Igf2-H19 locus (Figure 1, middle panel) leads to overexpression of $I g f$, which is observed in Beckwith-Wiedemann syndrome and is associated with organomegaly of newborns and a high incidence of several pediatric sarcomas (e.g. rhabdomyosarcoma and nephroblastoma) [18, 19].

In contrast, H19, as mentioned above, is a long, 2.3-kb, non-coding RNA (ncRNA) transcribed from the maternally inherited allele only; it is highly expressed prenatally and downregulated postnatally. It is well known that H19 ncRNA, which is evolutionarily conserved at the nucleotide level in humans and rodents and not translated to protein, is processed into small miRNAs [15]. This is supported by the finding that in situ hybridization of the H19 ncRNA revealed that it is detectable in cytoplasmic ribonucleoprotein particles, which suggests that H19-derived miRNAs are involved in ribosomal function and translation. However, the loss of $H 19$ is not lethal in mice, http://en.wikipedia.org/wiki/H19_(gene) and such animals display an overgrowth phenotype similar to babies with Beckwith-Wiedemann syndrome, while the overexpression of $\mathrm{H} 19$ is a dominant lethal mutation; mouse embryos overexpressing $\mathrm{H} 19$ die after embryonic day 14.

The close coupling of $I g f 2$ and $H 19$ expression is explained by the fact that these two genes share the same 3' gene enhancer (Figure 1). It has been reported that deletion of this 3' enhancer results in downregulation of both Igf 2 and $H 19$ expression. Furthermore, there are some indications that the 3' enhancer has a more robust effect on the expression of $H 19$ than Igf2. This could be explained by the facts that: 1) $H 19$ has a stronger promoter than Igf $2[4,14]$; and 2) that the $H 19$ gene is physically closer to the 3 ' enhancer than $\operatorname{Ig} 2$ (Figures 1 and 2).

The expression of Igf2-H19 is regulated from the 3 ' enhancer by the transcriptional zinc finger DNA-binding protein repressor, CTCF (also known as CCCTC-DNA sequence binding factor). The human genome contains nearly 15,000 CTCF insulator sites, suggesting a widespread role of CTCF in gene regulation $[5,14,16]$. What is of importance for this review is that this DNA-binding regulatory protein is involved in insulator activity for $I g f 2$ expression by binding to the DMR at the Igf-H19 locus (Figure 1). Binding to the methylation-free DMR by CTCF blocks the interaction between the 3' distal enhancer and the promoter of Igf2, while not affecting H19 expression. Overall, four CTCF binding sites have been identified in the murine Igf2-H19 DMR and seven in the human counterpart. Interestingly, the human Igf2-H19 DMR is not able to function when introduced as a transgene into the murine genome, which suggests differences in the regulation of this DMR between species [14].

In conclusion, proper imprinting of the Igf2-H19 locus is required for the balanced expression of both genes in somatic cells and in normal embryonic development.

\section{Biological consequences of erasure of imprinting at the DMR for the Igf2-H19 tandem gene in primordial germ cells (PGCs)}

The erasure of methylation in the DMR for Igf2-H19 (Figure 2, middle panel) leads to downregulation of growth-promoting $I g f 2$ from both paternal and maternal chromosomes and overexpression of proliferation-limiting H19. Therefore, cells that undergo such epigenetic modification tend to remain in a quiescent state, and this occurs early in the development of PGCs [6-11]. Accordingly, PGCs, while they migrate during embryogenesis through the embryo proper into the genital ridges, erase several maternally and paternally imprinted loci, including the Igf2-H19 locus. This mechanism of erasure of imprinted marks has several important consequences. First, it prevents these cells from uncontrolled proliferation and the potential risk of teratoma formation. Second, it creates in mammals a barrier against parthenogenetic development [4].

Furthermore, after the erasure of imprinting, PGCs are not able to proliferate in vitro and do not complement blastocyst development, while nuclei harvested from PGCs are not capable of performing as DNA donors in therapeutic cloning. However, all 
these limitations in the pluripotency of PGCs are reversed when their imprinting is re-established during ex vivo generation from PGCs of embryonic germ cells (EGCs) [6-11].

During normal development, proper imprinting in germ line cells is established in later stages when PGCs, after colonization of the genital ridges, develop into gametes - oocytes or sperm. Proper imprinting is established in female germ line (paternal imprinting) and male germ line (maternal imprinting) cells after a first meiotic division when the chromosomes are reduced to haploid number. Thus, when haploid gametes fuse during fertilization, the chromosomes in the diploid zygote have proper imprinting, including at the Igf2-H19 loci.

As mentioned above, the importance of proper imprinting of Igf2-H19 during embryogenesis has also been demonstrated during generation of viable bimaternal mice [20]. The bimaternal zygote is generated from two female sets of chromosomes without participation of a paternal genome by combining two haploid nuclei from non-growing oocytes - one derived from a newborn pup and another from a fully grown oocyte. Since female chromosomes have unmethylated DMRs for Igf2-H19, the proper expression of Igf 2 and $H 19$ in this mouse was achieved by appropriate genetic modification of the Igf2-H19 locus on one set of female chromosomes to ensure that this locus expresses $\operatorname{Ig} 2$ [20].

\section{Biological consequences of erasure of imprinting in the DMR of the Igf2-H19 tandem gene in very small embryonic-like stem cells (VSELs)}

Modification of genomic imprinting also plays a crucial role in maintaining the pool of pluripotent stem cells residing in adult tissues. Recently, our group demonstrated that adult murine tissues harbor a population of pluripotent Oct $4^{+}$SSEA- $1^{+}$Sca- $-1^{+}$Lin $^{-}$ CD45- cells [21, 22]. We have also identified a corresponding population of cells that are Oct- $4^{+}$SSEA$4^{+} \mathrm{CD} 133^{+} \mathrm{Lin}^{-} \mathrm{CD} 45^{-}$in humans as well $[23,24]$. We hypothesize that these PSCs, which are called VSELs, are deposited in adult tissues, including during early embryogenesis, and serve as a backup for tissue-committed stem cells (TCSCs). Molecular analysis of VSELs has revealed that their quiescence in adult BM and premature depletion from the tissues is controlled by epigenetic changes to imprinted genes, including the Igf2-H19 locus, which is erased in murine VSELs $[12,13]$ (Figure 2, middle panel).

Murine VSELs, in addition to erasure at the Igf2-H19 locus, also modify expression of other imprinted genes. For example, we observed that murine BM-sorted VSELs erase the paternally methylated imprints (e.g. DMRs at the Igf2-H19 and RasGrf1 loci, Figure 3), while they hypermethylate the maternally methylated imprints (e.g. DMRs at $I g f 2 R$ ). The epigenetic modification of imprinted loci (including Igf2-H19) prevents VSELs from uncontrolled proliferation and, as in the case of PGCs, prevents them from teratoma formation. The epigenetic modification of imprinted genes also explains why VSELs, despite expressing several markers of pluripotency (e.g. open chromatin structure at the promoters for Oct-4 and Nanog), the presence of bivalent domains at developmentally important homeobox-domain containing genes, the reactivation of the $\mathrm{X}$ chromosome in female VSELs, and in vitro differentiation into cells from all three germ layers, do not complement blastocyst development after injection into the pre-implantation blastocyst $[12,13]$.

There are also other important implications for erasure of paternally imprinted genes in VSELs. Namely, the erasure at the paternally imprinted Igf2-H19 and RasGrf1 loci leads to impair insulin/insulin-like growth factor signaling (IIS) in these cells [25-27]. This has a potential impact on the role of VSELs in tissue rejuvenation and aging as well as on the involvement of these cells in tumorogenesis.

\section{Erasure of imprinting at the Igf-H19 locus in VSELS and its implications for tissue rejuvenataion and aging}

The abovementioned changes in expression of imprinted genes in VSELs lead to perturbation of IIS by downregulation of IGF2, which is an autocrine factor involved in proliferation of VSELs, and downregulation of RasGRF1, which is a GTP-exchange factor (GEF) crucial for signaling from IGF-IR and the insulin receptor (InsR, Figure 3 ). In addition, in VSELs we observed hypermethylation of DMRs on the maternal chromosome encoding IGF-2R [12, 13], which, as mentioned above, serves as a decoy receptor that prevents IGF-2 from binding to IGF-IR [25]. Overall, this epigenetic reprogramming of genomic imprinting negatively affects IIS signaling, maintains the quiescent state of VSELs and thus protects them from premature depletion from the tissues, and prevents their involvement in tumor formation.

Based on these findings, we propose a novel hypothesis that relates aging, longevity, and IIS to the abundance and function of pluripotent VSELs deposited in adult tissues. A decrease in the number of these cells should negatively affect pools of TCSCs in various organs and have an impact on tissue rejuvenation and 


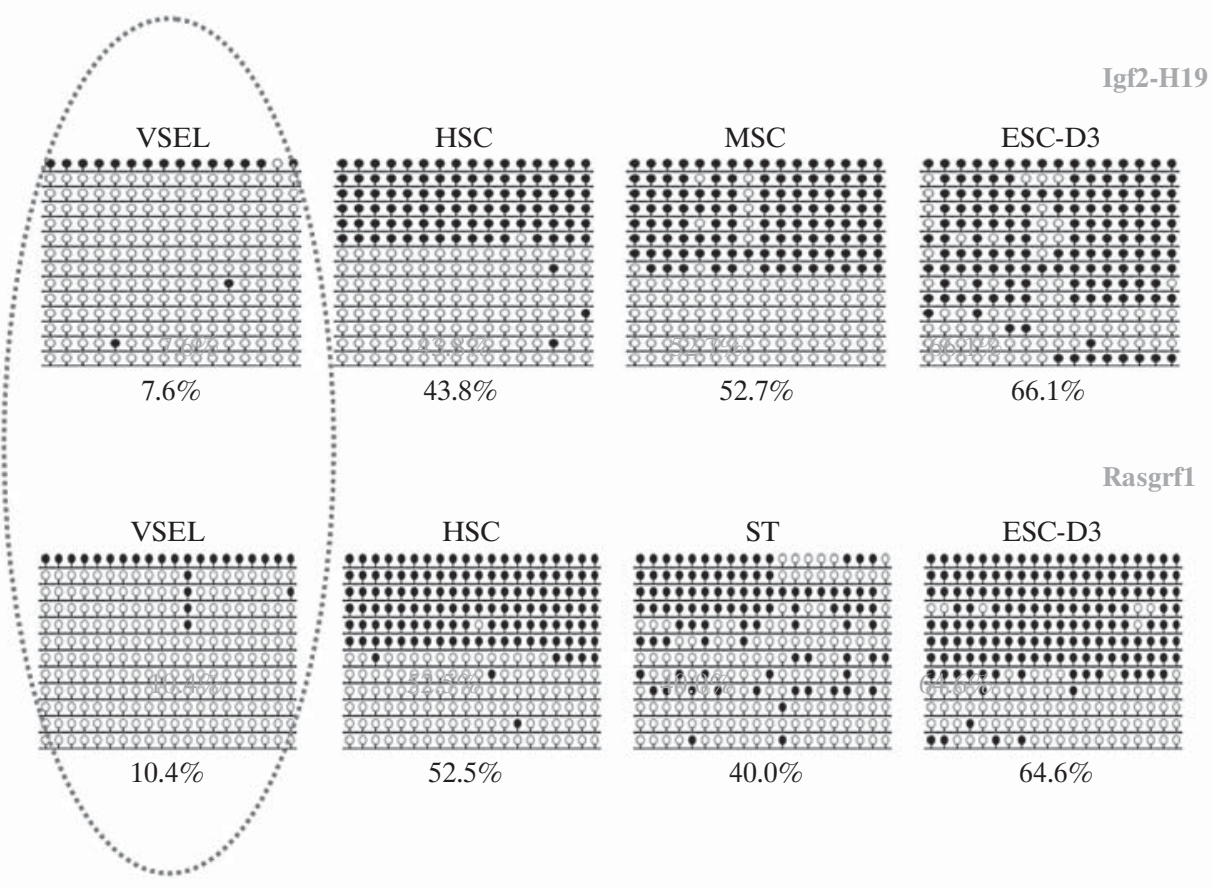

Figure 3. Erasure of genomic imprinting for paternally methylated imprinted genes in murine VSELs, HSCs, MSCs, and the ES-D3 cell line. Bisulfite sequencing profiles of DNA methylation of DMRs for Igf2-H19 (upper panel) and RasGrf1 (lower panel) loci in VSELs, hematopoietic stem cells (HSCs), mesenchymal stem cells (MSCs), and the murine embryonic stem cell line ES-D3. The percentage of methylated $\mathrm{CpG}$ sites was demonstrated by employing bisulfite modification and sequencing

lifespan [25-28]. In support of this expectation, we observed a significantly higher number of VSELs in long-living murine strains (e.g. Laron dwarfs and Ames dwarfs) whose longevity is explained by low levels of circulating IGF1 [29-32]. In contrast, the number of VSELs is reduced compared to normally aging littermates in mice with high levels of circulating IGF1 (e.g. growth hormone-overexpressing transgenic mice) [32].

Attempting to summarize all of this, the influence of IIS on the pool of VSELs residing in adult tissues seems to be twofold (Figure 4). In the first scenario, IGF2 is an autocrine factor for these cells, explaining why both downregulation of expression of $I g f 2$ and RasGrf1 together with elevated expression of $\mathrm{H} 19$ and upregulation of $I G F-2 R$, which is a decoy receptor for IGF2, keeps these cells quiescent. However, we observed that with increasing age, the methylation pattern of DMRs at paternally imprinted genes in VSELs reverses and Igf2-H19, as well as RasGrf1 loci, become gradually methylated, and thus VSELs become more sensitive to IIS [26-28]. This phenomenon may contribute to their age-related depletion over time.

On the other hand (second scenario), VSELs express functional IGF-1R and InsR and are susceptible to exogenous circulating IGF1 and insulin (Ins). Therefore, a chronic increase in caloric uptake that elevates circulating levels of these hormones may con- tribute over time to the depletion of these cells from adult tissues, affect the generation of VSEL-derived TCSCs, and thus negatively affect lifespan. In support of this scenario, we demonstrated that mice that have high levels of circulating blood plasma IGF1 show accelerated depletion of VSELs and have a shorter lifespan than age-matched littermates.

\section{Erasure of imprinting at the Igf-H19 locus in VSELs and its potential role in cancerogenesis}

The changes in imprinting of the Igf2-H19 locus may be involved in tumor formation. A growing body of evidence suggests that cancer originates in the stem/ /progenitor cell compartment. This stem-cell-origin-of-cancer hypothesis is based on the assumption that self-renewing stem cells residing in organs and tissues, and not mature differentiated somatic cells such as those lining the bronchial or stomach mucosa, may accumulate mutations over a lifetime [33, 34]. These mutations are subsequently maintained in stem cell compartments, and self-renewing stem cells may be subjected to additional mutations and epigenetic changes so that the genome is destabilized and uncontrolled neoplastic proliferation is initiated.

Interestingly, during the $19^{\text {th }}$ and early $20^{\text {th }}$ centuries, several investigators proposed that cancer de- 


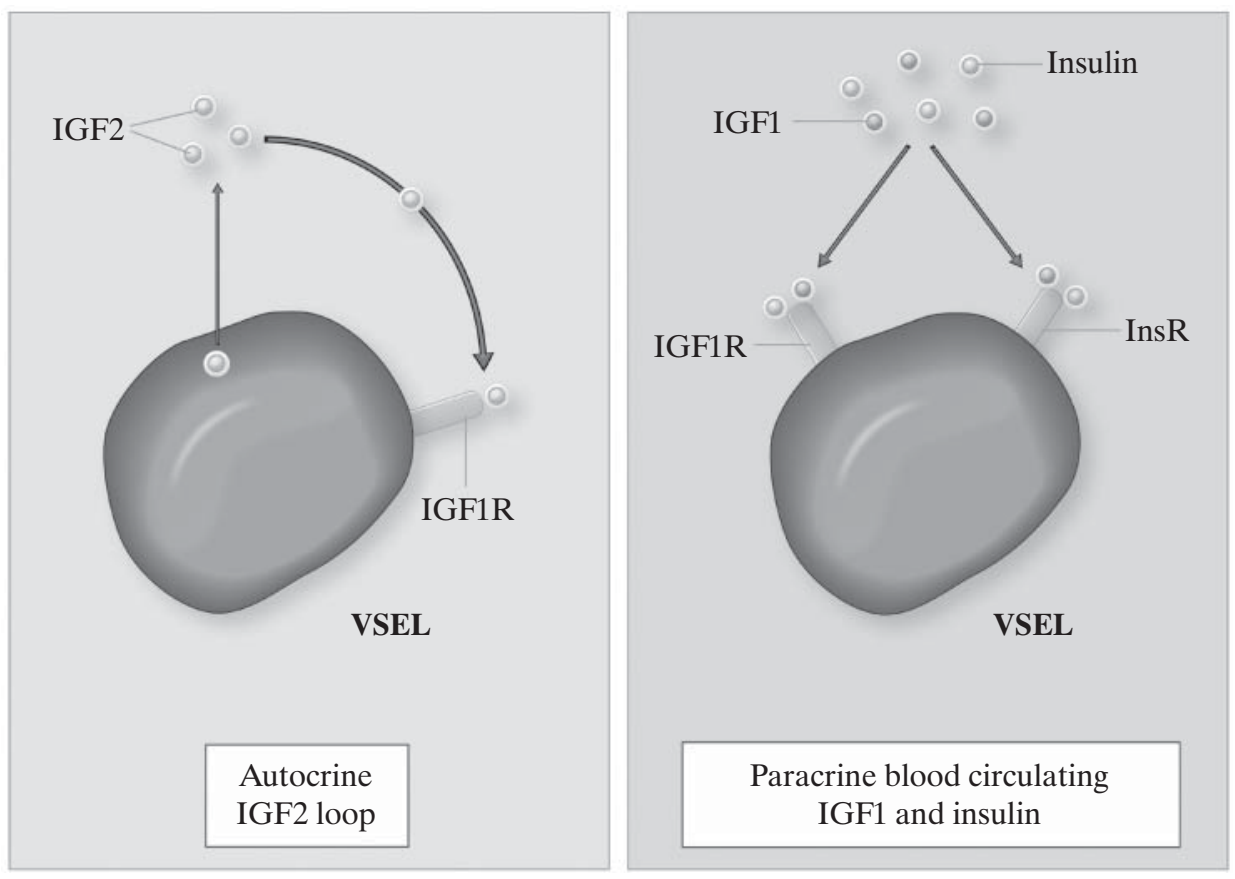

Figure 4. Two scenarios for how insulin/insulin-like growth factor signaling (IIS) may affect the pool of VSELs. VSELs are deposited in adult tissues as a backup population for tissue committed stem cells. Left panel — increase in autocrine-secreted IGF2, due to gradual loss of imprinting at the Igf2-H19 locus as observed during aging, stimulates VSELs via IGF-1R. This leads to an age-mediated VSEL depletion, and as a consequence a decrease in VSEL-generated tissue-committed stem cells (TCSCs), and impairs tissue organ rejuvenation. Right panel — VSELs deposited in adult tissues may, over time, become depleted more quickly by chronic elevated circulating levels of IGF1 and insulin that engage IGF1R and InsR expressed by these cells. This may contribute to accelerated aging observed in situations with high circulating IGF1 and insulin level (e.g. high calorie uptake). On other hand, this mechanism of chronic exposure to IIS may also lead to malignant transformation of VSELs after prolonged stimulation by IGF1 and insulin. For the sake of simplicity, two other proteins encoded by imprinted genes that affect IIS are not shown - paternally imprinted RasGrf1, which due to erasure of imprinting is not expressed in VSELs, and maternally imprinted $I G F-2 R$, which due to hypermethylation of the DMR locus on the maternal chromosome, is highly expressed in these cells. While RasGRF1 is an important component of IIS from activated IGF-1R and InsR, IGF-2R serves as a decoy receptor for IGF2. Therefore, changes in expression of these genes in VSELs further decrease the responsiveness of these cells to IIS

velops in populations of cells that are left in a dormant state in developing organs during embryogenesis [34]. This 'embryonic rest' hypothesis of cancer origin suggested that adult tissues contain embryonic remnants that normally lie dormant, but can be activated to become cancerous. Based on the presence of VSELs in adult tissues, we hypothesize that VSELs could be the missing link that reconciles the embryonic rest hypothesis of cancer origin with current theories envisioning cancer as a stem cell disorder.

In support of this perspective, it is well known that obesity and high caloric uptake are risk factors for cancer development. Experimental animals with high levels of circulating IGF1 are not only short-lived but also have a high incidence of cancer [35]. On the other hand, long-living animals with low levels of circulating IGF2 have a much lower incidence of tumor growth [32]. This correlates with human Laron dwarfs that have a very low level of circulating IGF1 and at the same time are prone to cancer development [29,
32]. Based on these observations, we envision that chronic stimulation of VSELs by IIS may activate these cells in an uncontrolled way and promote their malignant growth. Therefore, it is likely that some human tumors originate in VSELs, and that IIS may play an important initiating role. Again, we envision here two possible mechanisms. Firstly VSELs exposed to a constant high circulating IGF1 level could transform into neoplastic cells; and secondly, as discussed below, they could transform due to a loss of imprinting at the Igf2-H19 locus that will expose them to autocrine IGF2.

\section{Consequences for tumorogenisis of the loss of imprinting at the DMR for the Igf2-H19 tandem gene}

There is growing evidence that the autocrine IGF2-IGF-1R loop, due to loss of imprinting at the Igf2-H19 locus (Figure 2, lower panel), may be involved 
in the growth of several types of tumors. The best example, as mentioned above, is Beckwith-Wiedemann syndrome, which is associated with the development of several pediatric sarcomas. The loss of imprinting at the Igf2-H19 locus is a very well-known phenomenon observed both in Beckwith-Wiedemann syndrome-related and spontaneous human rhabdomyosarcomas and nephroblastomas. Loss of imprinting at this locus has also been described in gastrointestinal tumors, both in humans and in mice [36-39].

Furthermore, as mentioned above, one of the features of aging VSELs is gradual hypermethylation of DMRs for Igf2-H19 locus on the maternal chromosome that due to this 'age-related' loss of imprint leads to overexpression of IGF2 [26-28]. This may correspond to a higher incidence of cancer in advanced age. The deregulation of the Igf2-H19 locus observed in several tumors raises the question of the cell of origin in which this genetic defect originates. As discussed above, one possibility is that this event occurs in VSELs [33, 34]. This hypothesis, however, requires further studies.

\section{Conclusions}

Evidence has accumulated that the imprinted Igf2$H 19$ tandem gene plays a pleiotropic role in several biological processes. Expression of this locus is tightly regulated by genomic imprinting and the $\mathrm{CTCF}$ protein, which is involved in the balanced expression of both genes from either paternal or maternal chromosomes. In addition, an interesting mechanism has been described in which elevated levels of IGF2 in senescent human epithelial cells are the result of a reduction in CTCF expression, which controls the Igf2-H19 locus. As reported, an increase in the intracellular CTCF level, leading to lower occupancy of DMRs by CTCF within Igf-H19 loci, resulted in a 10 -fold increase in intracellular Igf 2 expression [14].

Furthermore, it is likely that, in addition to CTCF, other proteins are also involved in the regulation of this locus and that they still await identification. Modification of expression at the Igf2-H19 locus may have an important role in inhibiting aging processes and preventing cancerogenesis. Furthermore, as we envision, proper methylation of the DMR at this locus, which is erased in VSELs [12, 13], will be crucial for the development of ex vivo strategies for the expansion of VSELs for the purposes of regenerative medicine [40].

\section{Acknowledgments}

This work was supported by NIH grant R01 DK074720, the Stella and Henry Endowment, and the European
Union structural funds (Innovative Economy Operational Program POIG.01.01.02-00-109/09).

\section{References}

1. Reik W, Walter J. Genomic imprinting: parental influence on the genome. Nat Rev Genet. 2001;2:21-32.

2. Pannetier Ml, Feil R. Epigenetic stability of embryonic stem cells and developmental potential. Trends in Biotechnology. 2007;25:556-562.

3. Delaval K, Feil R. Epigenetic regulation of mammalian genomic imprinting. Curr Opin Genet Dev. 2004;14:188-195.

4. Bartolomei MS, Ferguson-Smith AC. Mammalian genomic imprinting. Cold Spring Harbor Perspect Biol. 2011;3:a002592

5. Pant V, Mariano $\mathrm{P}$, Kanduri $\mathrm{C}$ et al. The nucleotides responsible for the direct physical contact between the chromatin insulator protein CTCF and the H19 imprinting control region manifest parent of origin-specific long-distance insulation and methylation-free domains. Genes Dev. 2003;17:586-590.

6. Matsui Y, Zsebo K, Hogan BLM. Derivation of pluripotential embryonic stem cells from murine primordial germ cells in culture. Cell. 1992;70:841-847.

7. Yamazaki Y, Mann MR, Lee SS et al. Reprogramming of primordial germ cells begins before migration into the genital ridge, making these cells inadequate donors for reproductive cloning. Proc Natl Acad Sci USA. 2003;100:12207-12212.

8. Hajkova $\mathrm{P}$, Erhardt $\mathrm{S}$, Lane $\mathrm{N}$ et al. Epigenetic reprogramming in mouse primordial germ cells. Mech Dev. 2002;117:15-23.

9. Shovlin TC, Durcova-Hills G, Surani A et al. Heterogeneity in imprinted methylation patterns of pluripotent embryonic germ cells derived from pre-migratory mouse germ cells. Dev Biol. 2008;313:674-681.

10. Durcova-Hills G, Tang F, Doody G et al. Reprogramming Primordial Germ Cells into Pluripotent Stem Cells. PLoS ONE. 2008;3:e3531.

11. Lefebvre L, Viville S, Barton SC et al. Abnormal maternal behaviour and growth retardation associated with loss of the imprinted gene Mest. Nat Genet. 1998;20:163-169.

12. Shin DM, Zuba-Surma EK, Wu W et al. Novel epigenetic mechanisms that control pluripotency and quiescence of adult bone marrow-derived Oct- $4^{+}$very small embryonic like stem cells. Leukemia. 2009;23:2042-2051.

13. Shin DM, Liu R, Klich I et al. Molecular signature of adul bone marrow-purified very small embryonic-like stem cells supports their developmental epiblast/germ line origin. Leukemia. 2010;24:1450-1461.

14. Fu VX, Schwarze SR, Kenowski ML et al. A loss of insulinlike growth factor-2 imprinting is modulated by CCCTC-binding factor down-regulation at senescence in human epithelial cells. J Biol Chem. 2004;279:52218-52226.

15. Gabory A, Jammes H, Dandolo L. The H19 locus: role of an imprinted non-coding RNA in growth and development. Bioessays. 2010;32:473-480.

16. Yoon B, Herman H, Hu B et al. Rasgrf1 Imprinting Is Regulated by a CTCF-Dependent Methylation-Sensitive Enhancer Blocker. Mol Cell Biol. 2005;25:11184-11190.

17. Ratajczak MZ, Kucia M, Liu R et al. RasGrf1: genomic imprinting, VSELs, and aging. Aging. 2011;3:692-697.

18. Choufani S, Shuman C, Weksberg R. Beckwith-Wiedemann syndrome. Am J Med Genet C Semin Med Genet. 2010; 154C:343-354.

19. Eggenschwiler J, Ludwig T, Fisher P et al. Mouse mutant embryos overexpressing IGF-II exhibit phenotypic features of the Beckwith-Wiedemann and Simpson-Golabi-Behmel syndromes. Genes Dev. 1997;11:3128-3142. 
20. Kono T, Obata $\mathrm{Y}, \mathrm{Wu} \mathrm{Q}$ et al. Birth of parthenogenetic mice that can develop to adulthood. Nature. 2004;428:860-864 .

21. Kucia M, Reca R, Campbell FR et al. A population of very small embryonic-like (VSEL) CXCR4+SSEA-1+Oct-4+ stem cells identified in adult bone marrow. Leukemia. 2006;20:857-869.

22. Zuba-Surma EK, Kucia M, Ratajczak J et al. "Small stem cells" in adult tissues: Very Small Embryonic-like Stem Cells (VSELs) stand up! Cytometry. 2009;75A:4-13.

23. Wojakowski W, Tendera M, Kucia M et al. Mobilization of Bone Marrow-Derived Oct- $4^{+}$SSEA-4 ${ }^{+}$Very Small Embryonic-Like Stem Cells in Patients with Acute Myocardial Infarction. J Am Coll Cardiol. 2009;53:1-9.

24. Paczkowska E, Kucia M, Koziarska D et al. Clinical evidence that very small embryonic-like (VSEL) stem cells are mobilized into peripheral blood in patients after stroke. Stroke. 2009;40:1237-1244.

25. Ratajczak MZ, Shin DM, Ratajczak J et al. A novel insight into aging: are there pluripotent very small embryonic-like stem cells (VSELs) in adult tissues overtime depleted in an IGF-1-dependent manner? Aging (Albany NY). 2010;2:875-883.

26. Ratajczak J, Shin DM, Wan W et al. Higher number of stem cells in bone marrow of circulating Igf-1 level low Laron dwarf mice - novel view on Igf-1, stem cells and aging. Leukemia. 2011;25:729-733.

27. Kucia M, Shin DM, Liu R et al. Reduced number of VSELs in bone marrow of Growth Hormone transgenic mice indicates that chronically elevated Igf- 1 level accelerates age-dependent exhaustion of pluripotent stem cell pool - novel view on aging. Leukemia. 2011;25:1370-1374.

28. Kucia M, Masternak M, Liu R et al. The negative effect of prolonged somatotrophic/insulin signaling on an adult bone marrow-residing population of pluripotent very small embryonic-like stem cells (VSELs).Age. 2012 (doi: 10.1007/s11357-011-9364-8).

29. Steger RW, Bartke A, Cecim M. Premature ageing in transgenic mice expressing different growth hormone genes. $J$ Reprod Fertil Suppl. 1993;46:61-75.
30. Kucia M, Ratajczak J, Ratajczak MZ. Are bone marrow stem cells plastic or heterogenous - that is a question. Exp. Hematol. 2005;33:613-623.

31. Bartke A, Brown-Borg H. Life extension in the dwarf mouse. Curr Top Dev Biol. 2004;63:189-225.

32. Bartke A, Chandrashekar V, Bailey B et al. Consequences of growth hormone $(\mathrm{GH})$ overexpression and $\mathrm{GH}$ resistance. Neuropeptides. 2002;36:201-208.

33. Ratajczak MZ, Dong-Myung Shin, Kucia M. Very small embryonic-like stem cells (VSELs): a missing link to support the germ line hypothesis of cancer development? Am J Pathol. 2009;174:1985-1992.

34. Ratajczak MZ, Shin DM, Liu R et al. Epiblast/Germ Line Hypothesis of Cancer Development Revisited: Lesson from the Presence of Oct- $4^{+}$Cells in Adult Tissues. Stem Cell Rev \& Rep. 2010;6:307-316.

35. Guevara-Aguirre J, Balasubramanian P, Guevara-Aguirre M et al. Growth Hormone Receptor Deficiency Is Associated with a Major Reduction in Pro-Aging Signaling, Cancer, and Diabetes in Humans. Science Transl Med. 2011;70:70ra13.

36. Nakagawa N, Chadwick RB, Peltomaki P et al. Loss of imprinting of the insulin-like growth factor II gene occurs by biallelic methylation in a core region of H19-associated CTCF-binding sites in colorectal cancer. Proc Natl Acad Sci USA. 2001;98:591-596.

37. Sakatani T, Kaneda A, Iacobuzio-Donahue CA et al. Loss of Imprinting of Igf2 Alters Intestinal Maturation and Tumorigenesis in Mice. Science. 2005;307:1976-1978.

38. Fu VX, Dobosy JR, Desotelle JA et al. Aging and Cancer-Related Loss of Insulin-like Growth Factor 2 Imprinting in the Mouse and Human Prostate. Cancer Res. 1999;59:3889$-3892$.

39. Rainier S, Dobry CJ, Feinberg AP. Loss of Imprinting in Hepatoblastoma. Cancer Res. 1995;55:1836-1838.

40. Ratajczak MZ, Kucia M, Jadczyk T et al. Pivotal Role of Paracrine Effects in Stem Cell Therapies in Regenerative Medicine - Can We Translate Stem Cell-Secreted Paracrine Factors and Microvesicles into Better Therapeutic Strategies? Leukemia. 2011 epub doi: 10.1038/leu.2011.389.

Submitted: 25 January, 2012 Accepted after reviews: 22 February, 2012 